\section{Precisión diagnóstica de indicadores antropométricos: perímetro de cintura, índice cintura-talla e índice cintura-cadera para la identificación de sobrepeso y obesidad infantil}

\author{
Saldívar-Cerón $\mathrm{HI}^{1}$, Vázquez-Martínez $\mathrm{AL}^{1}$, Barrón-Torres $\mathrm{MT}^{2}$
}

\section{Resumen}

INTRODUCCIÓN: la identificación de obesidad durante la infancia es complicada debido a que la talla sigue incrementándose y la composición corporal está cambiando constantemente; actualmente su diagnóstico se realiza por medio de los patrones de crecimiento propuestos por la Organización Mundial de la Salud (OMS); sin embargo, se han propuesto diversos indicadores antropométricos para una detección equivalente.

OBJETIVO: determinar la precisión diagnóstica del perímetro de cintura, el índice cintura-talla y el índice cintura-cadera para la identificación de sobrepeso y obesidad infantil.

MATERIAL Y MÉTODOS: estudio transversal y analítico con una muestra de 1,001 escolares (9-11 años) de ambos géneros. Se hicieron las mediciones antropométricas (peso, talla, perímetro de cintura y perímetro de cadera) y se calculó el índice de masa corporal, índice cintura-talla e índice cintura-cadera. Se evaluó la correlación de los índices antropométricos vs. el índice de masa corporal y se efectuó un análisis de curvas ROC tomando como variable de estudio el perímetro de cintura, índice cintura-talla e índice cintura-cadera y como parámetro de referencia los percentiles de índice de masa corporal (OMS, 2007). Se obtuvieron la sensibilidad, especificidad, las áreas bajo la curva, el valor predictivo positivo y negativo de los indicadores antropométricos y los puntos de corte que identifican sobrepeso y obesidad.

RESULTADOS: el perímetro de cintura y el índice cintura-talla tienen una alta correlación positiva $(r=0.75,0.69$, respectivamente; $\mathrm{p}<0.001)$, pero la correlación fue débil para el índice cintura-cadera $(r=0.23 ; \mathrm{p}<0.001)$. El perímetro de cintura e índice cintura-talla mostraron grandes áreas bajo la curva, sensibilidad, especificidad, valor predictivo positivo y valor predictivo negativo para la identificación de obesidad infantil.

CONCLUSIÓN: el perímetro de cintura y el índice cintura-talla tienen un alto poder predictivo para identificar obesidad infantil, no así para sobrepeso. Los puntos de corte sugeridos para el diagnóstico de obesidad infantil entre 9 y 11 años son de $70 \mathrm{~cm}$ para perímetro de cintura y 0.5 para índice cintura-talla en ambos géneros.

PALABRAS CLAVE: obesidad infantil, precisión diagnóstica, epidemiología.
${ }^{1}$ Facultad de Estudios Superiores Cuautitlán, Universidad Nacional Autónoma de México, Cuautitlán Izcalli, Estado de México, México. ORCID iD: http:// orcid.org/0000-0002-9125-9100

2Facultad de Medicina de Tampico Dr. Alberto Romo Caballero, Universidad Autónoma de Tamaulipas, Tampico, Tamaulipas, México.

Recibido: 17 de septiembre del 2015

Aceptado: 2 de febrero del 2016

Correspondencia

Ana Laura Vázquez Martínez

Facultad de Estudios Superiores Cuautitlán Universidad Nacional Autónoma de México Cuautitlán Izcalli, Estado de México, México. analaumex@hotmail.com

Este artículo debe citarse como Saldívar-Cerón HI, Vázquez-Martínez AL, BarrónTorres MT. Precisión diagnóstica de indicadores antropométricos: perímetro de cintura, índice cintura-talla e índice cintura-cadera para la identificación de sobrepeso y obesidad infantil. Acta Pediatr Mex. 2016;37(2):79-87. 


\section{Diagnostic accuracy of anthropometric indicators: waist circumference, waist- height index and waist-hip ratio to identify overweight and childhood obesity}

\author{
Saldívar-Cerón $\mathrm{HI}^{1}$, Vázquez-Martínez $\mathrm{AL}^{1}$, Barrón-Torres $\mathrm{MT}^{2}$
}

\section{Abstract}

INTRODUCTION: The identification of obesity in childhood is complicated because the height continues to increase and body composition is constantly changing, the diagnosis is currently done through growth patterns proposed by the WHO, however diverse anthropometric indicators have been proposed for equivalent detection.

OBJECTIVE: To determinate the diagnostic accuracy of waist circumference (WC), waist-to-height ratio (WHtR) and waist-hip ratio (WHR) for identification of childhood overweight and obesity.

MATERIAL AND METHODS: A cross-sectional and analytical study with a sample of 1001 schoolchildren (aged 9 to 11-years). Anthropometric measurements of weight, height, WC and hip circumference were obtained. BMI, WHtR and WHR were calculated. The correlation of anthropometric indexes vs BMI was assessed and a ROC curve analysis was performed taking as a study variable: WC, WHtR and WHR and as baseline the BMI percentiles (WHO, 2007). Sensitivity (Se), specificity $(S p)$, areas under the curve (AUC), positive and negative predictive values (PPV, NPV) of anthropometric indicators and cutoffs points that identify overweight and obesity were obtained.

RESULT: The WC and WHtR showed strong positive correlation ( $r=0.75$, 0.69 , respectively; $p<0.001)$, but the correlation was weak for the WHR $(r=0.23 ; p<0.0001)$. WC and WHtR showed high ABC, Se, Sp, PPV and NPV for the identification of childhood-obesity.

CONCLUSION: WC and WHtR has a high predictive power to identify childhood-obesity but not for overweight. The suggested cut-off points for the diagnosis of childhood-obesity between 9 and 11 years are $70 \mathrm{~cm}$ for WC and 0.5 for ICT for both genders.

KEY WORDS: Childhood obesity; diagnostic accuracy; epidemiology
${ }^{1}$ Facultad de Estudios Superiores Cuautitlán, Universidad Nacional Autónoma de México, Cuautitlán Izcalli, Estado de México, México. ORCID iD: http://orcid.org/0000-00029125-9100

2Facultad de Medicina de Tampico Dr. Alberto Romo Caballero, Universidad Autónoma de Tamaulipas, Tampico, Tamaulipas, México.

Correspondence

Ana Laura Vázquez Martínez

Facultad de Estudios Superiores Cuautitlán Universidad Nacional Autónoma de México Cuautitlán Izcalli, Estado de México, México.

analaumex@hotmail.com

\section{INTRODUCCIÓN}

La obesidad se define como una condición de acumulación anormal o excesiva de grasa en el tejido adiposo (adiposidad) que es perjudicial para la salud. La identificación de obesidad durante la infancia es complicada debido a que la talla sigue incrementando y la composición 
corporal está cambiando continuamente. ${ }^{1}$ Sin embargo, existen métodos de detección directa de adiposidad (resonancia magnética, tomografía, densitometría y la absorciometría con rayos $X$ de doble energía) y son considerados mejores que los métodos indirectos, como el índice de masa corporal, el cual no puede distinguir entre tejido adiposo y el crecimiento de otros tejidos; empero, los métodos directos son muy costosos y complicados de llevar a cabo y no están recomendados para la práctica clínica de rutina. ${ }^{2-4}$

En la consulta de primer nivel el diagnóstico de obesidad infantil se realiza por medio de los patrones de crecimiento de la Organización Mundial de la Salud 2007, particularmente por los percentiles de índice de masa corporal para el género y edad. ${ }^{5,6}$ No obstante, existen diversos indicadores antropométricos propuestos para el diagnóstico de sobrepeso y obesidad infantil como el perímetro de cintura, el índice cinturatalla y el índice cintura-cadera sin necesidad de calcular el índice de masa corporal; pese a esto, el método indirecto ideal debe ser barato, sensible, preciso, rápido y bien documentado., ${ }^{2,-9}$

\section{OBJETIVO}

Evaluar la precisión diagnóstica y los puntos de corte de los siguientes indicadores antropométricos: perímetro de cintura, índice cintura-talla e índice cintura-cadera para la identificación de sobrepeso y obesidad infantil.

\section{MATERIAL Y MÉTODOS}

Estudio analítico, prolectivo y transversal con una muestra total de 1,128 escolares en serie consecutiva y sin hacer distinción entre los participantes, con edades comprendidas entre los 9 y 11 años. Se excluyeron 127 escolares por presentar bajo peso, trastornos endocrinológicos o sistémicos previamente conocidos como hipotiroidismo, diabetes mellitus tipo 1 etc., quedando un grupo final constituido por 1,001 individuos (Figura 1).

El estudio fue aprobado por el Comité de Ética de la Facultad de Medicina de Tampico y la Universidad Autónoma de Tamaulipas con registro: FMT-SA-008/11. Se obtuvo el consentimiento informado firmado de los padres, respetando la normativa de Helsinki.

Los datos fueron recopilados durante los meses de enero a mayo de 2014 en 14 escuelas públicas de la ciudad de Tampico, Tamaulipas; seleccionadas de manera aleatoria y forman parte de manera secundaria de un estudio recientemente publicado sobre la obesidad infantil y el pie plano. ${ }^{10}$

En todos los participantes se realizaron mediciones antropométricas (peso, talla, perímetros de cintura y cadera) por métodos convenciona-

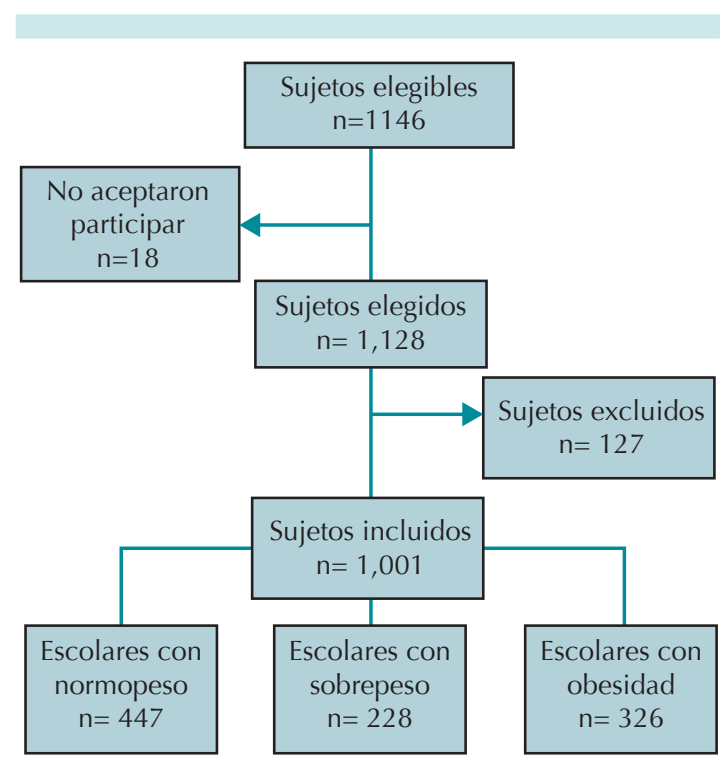

Figura 1. Diagrama de flujo de los participantes del estudio. Se excluyeron 127 escolares por presentar bajo peso, trastornos endocrinológicos o sistémicos previamente conocidos como hipotiroidismo, diabetes mellitus tipo 1, etc. 
les (utilizando una báscula mecánica de piso calibrada y una cinta métrica flexible) y estandarizados por personal previamente capacitado para la recolección de datos, el mismo día de la cita y sin incluir ningún tipo de tratamiento.

\section{Descripción de las variables}

Las variables consideradas para este estudio fueron: edad, género, talla, índice de masa corporal, índice cintura-talla, perímetro de cintura e índice cintura-cadera. El peso fue determinado al valor más cercano a $0.1 \mathrm{~kg}$ y la talla al valor más cercano a $0.5 \mathrm{~cm}$ con el escolar en posición de bipedestación, con ropa ligera sin calzado, en espiración gentil. El perímetro de cintura fue medido tomando el punto medio entre la última costilla y el borde superior de la cresta ilíaca, la medición de la circunferencia de la cadera se realizó en el punto más prominente de los glúteos coincidiendo con la sínfisis del pubis. ${ }^{11}$

A partir de estas mediciones se calculó el índice de masa corporal usando el índice de Quetelet: peso en kilogramos divido por el cuadrado de la talla en metros $\left(\mathrm{kg} / \mathrm{m}^{2}\right)$, índice cintura-cadera (cociente entre el perímetro de la cintura sobre el perímetro de la cadera en centímetros) y el índice cintura-talla (resultado de la división del perímetro de la cintura en centímetros sobre la talla en centímetros).

Posteriormente, cada escolar fue categorizado a partir de las tablas de percentiles de índice de masa corporal para género y edad de la OMS 2007 , en normopeso $(P>5-<85)$, sobrepeso (P85 - <95), obesidad (P>95), los escolares con bajo peso $(\mathrm{P}<5)$ fueron eliminados del estudio.

\section{Análisis estadístico}

Los datos están representados por la media \pm desviación estándar (DE). El análisis estadístico se realizó con los programas SPSS v.20 y Graph
Pad Prism 6. Se realizó una prueba ANOVA de una vía para evaluar el comportamiento de las variables antropométricas durante el periodo de crecimiento. Se determinó el coeficiente de correlación de Pearson para estimar la relación entre el índice de masa corporal y los diferentes métodos de identificación de sobrepeso y obesidad. Se efectuó un análisis de curvas ROC (Receiver Operating Characteristics) para evaluar la sensibilidad y especificidad del índice cintura-talla, el perímetro de cintura y el índice cintura-cadera para la detección de sobrepeso y obesidad infantil. Se utilizaron como parámetros de referencia las categorías nutricionales (normopeso, sobrepeso y obesidad) establecidas mediante los percentiles de índice de masa corporal, considerando significancia estadística $\mathrm{p}<0.05$, con intervalo de confianza (IC) de 95\%. Se utilizó como punto de corte el nivel de la variable que maximiza de forma conjunta la sensibilidad y especificidad de los métodos de detección para sobrepeso y obesidad, además se determinaron sus valores predictivos positivos (VPP) y negativos (VPN).

\section{RESULTADOS}

La muestra final estuvo constituida por 1,001 escolares. Las medidas antropométricas se encuentran resumidas en el Cuadro 1. Las medias del peso, talla, índice de masa corporal (IMC) y perímetro de cintura (PC) comparados por medio de ANOVA difirieron entre los géneros exceptuando el índice cintura-talla (ICT) e índice cintura-cadera (ICC) que permanecieron estables. El percentil >95 para el IMC fue del 33.9\% para varones y $24.1 \%$ para mujeres.

Todos los indicadores antropométricos correlacionaron positivamente con el IMC $(p<0.001)$. El coeficiente de correlación del PC fue alto en ambos géneros (hombres: 0.75, mujeres 0.74), de igual forma el ICT; sin embargo, el ICC presentó una correlación baja (hombres 0.23 , mujeres 0.16) Cuadro 2. 
Saldívar-Cerón HI et al. Indicadores antropométricos de obesidad infantil

Cuadro 1. Medidas antropométricas clasificadas por edad y género

\begin{tabular}{|c|c|c|c|c|c|c|c|}
\hline Edad (años) & $\mathbf{n}$ & Peso (kg)* & Talla $(\mathbf{m})^{*}$ & IMC* & ICT & $\mathrm{PC}(\mathbf{c m})^{*}$ & ICC \\
\hline & & Media $\pm \mathrm{DE}$ & Media \pm DE & Media $\pm \mathrm{DE}$ & Media \pm DE & Media $\pm \mathrm{DE}$ & Media $\pm \mathrm{DE}$ \\
\hline \multicolumn{8}{|l|}{ Hombres } \\
\hline 9 & 124 & $37.5 \pm 9.7$ & $1.37 \pm 0.06$ & $19.7 \pm 3.9$ & $0.50 \pm 0.05$ & $69 \pm 9.4$ & $0.9 \pm 0.04$ \\
\hline 10 & 239 & $41.7 \pm 11.4$ & $1.40 \pm 0.07$ & $20.7 \pm 4.4$ & $0.50 \pm 0.07$ & $71 \pm 12.3$ & $0.8 \pm 0.1$ \\
\hline 11 & 132 & $48.1 \pm 12.9$ & $1.47 \pm 0.08$ & $21.7 \pm 4.4$ & $0.50 \pm 0.08$ & $74 \pm 14.0$ & $0.8 \pm 0.1$ \\
\hline \multicolumn{8}{|l|}{ Mujeres } \\
\hline 9 & 143 & $37.8 \pm 9.8$ & $1.37 \pm 0.07$ & $19.7 \pm 3.8$ & $0.49 \pm 0.05$ & $68 \pm 8.5$ & $0.8 \pm 0.04$ \\
\hline 10 & 219 & $42.3 \pm 10.5$ & $1.43 \pm 0.07$ & $20.4 \pm 4.1$ & $0.48 \pm 0.07$ & $69.3 \pm 12.4$ & $0.8 \pm 0.1$ \\
\hline 11 & 144 & $46.9 \pm 11.7$ & $1.48 \pm 0.07$ & $21.1 \pm 4.4$ & $0.48 \pm 0.07$ & $71.3 \pm 11.1$ & $0.8 \pm 0.1$ \\
\hline
\end{tabular}

IMC: índice de masa corporal; ICT: índice cintura-talla; PC: perímetro de cintura; ICC: índice cintura-cadera. ANOVA de una vía para evaluar el comportamiento de las variables antropométricas durante el periodo de crecimiento * $\mathrm{p}<0.01$.

Cuadro 2. Coeficiente de correlación bi-variadas entre los métodos de identificación

\begin{tabular}{lcc}
\hline & \multicolumn{2}{c}{ IMC $\left(\mathbf{k g} / \mathbf{m}^{2}\right)$} \\
& Hombres $(\mathbf{n}=\mathbf{4 9 5})$ & Mujeres $(\mathbf{n = 5 0 6})$ \\
PC $(\mathrm{cm})$ & 0.75 & 0.74 \\
ICT & 0.69 & 0.68 \\
ICC & 0.23 & 0.16
\end{tabular}

IMC: índice de masa corporal; PC: perímetro de cintura; ICT: índice cintura-talla; ICC: índice cintura-cadera. Todas las correlaciones fueron significativas $(p<0.001)$.

Las curvas ROC para sobrepeso y obesidad se muestran en la Figura 2. El poder discriminativo para la identificación de obesidad a través del PC (área bajo la curva [ABC] de 0.96 en ambos sexos) fue alto. Muy cercano se encuentra el ICT ( $A B C$ de 0.94 en hombres y 0.95 en mujeres) y en no tan connotada posición el ICC ( $\mathrm{ABC}$ de 0.71 en hombres y 0.64 en mujeres) Cuadro 3.

El punto de corte de $70 \mathrm{~cm}$ para el PC tiene un gran poder discriminativo en ambos géneros (hombres: sensibilidad [S]: $91.4 \%$, especificidad [E]: 92.2\%, valor predictivo positivo [VPP]: 93\%, valor predictivo negativo [VPN] 91\%; mujeres (S) 92.1\%, E 91\%, VPP: 95\%, VPN $87 \% ; p<0001)$. Para el ICT el punto de corte fue de 0.50 para ambos géneros (hombres $S$ : 87.7\%, E: 96.1\%, VPP: 94 VPN: 94; mujeres: S 83.4\%, E 95.1\% VPP: 95 VPN: 93). El ICC no tiene poder discriminativo para la identificación de obesidad.

En el caso de sobrepeso, el poder discriminativo del PC, ICT e ICC en ambos géneros fue menor que para obesidad, obteniendo $\mathrm{ABC}$ por debajo de 0.85 , con $\mathrm{S}$ y $\mathrm{E}<85 \%$, VPP menor a $50 \%$ (Cuadro 4).

\section{DISCUSIÓN}

Determinar los valores más apropiados para la evaluación del crecimiento infantil en una población es complicado. En México son pocos los estudios sobre el crecimiento de la población pediátrica a diferencia de España, la cual tiene una larga tradición de estudios de crecimiento. Entre los más populares se encuentran las curvas y tablas de la Fundación Faustino Osbergozo. Estos resultados han sido tomados como referencia para el diagnóstico de obesidad infantil en población latinoamericana; sin embargo, la necesidad de tener valores de referencia internacionales impulsaron a la OMS a realizar un estudio multicéntrico sobre crecimiento, el cual fue efectuado en población pediátrica de Brasil, EEUU, Ghana, India, Noruega y Omán, entre el 2004-2007. A partir de este estudio se publicaron los puntajes z y percentiles del 
PC

Hombres

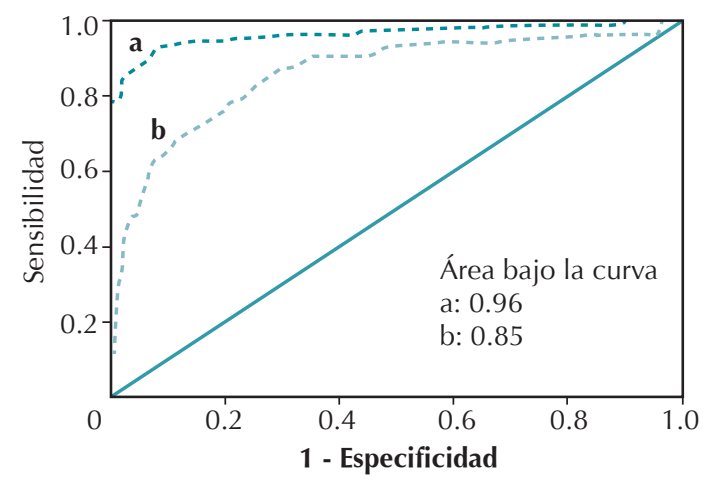

ICT

Hombres

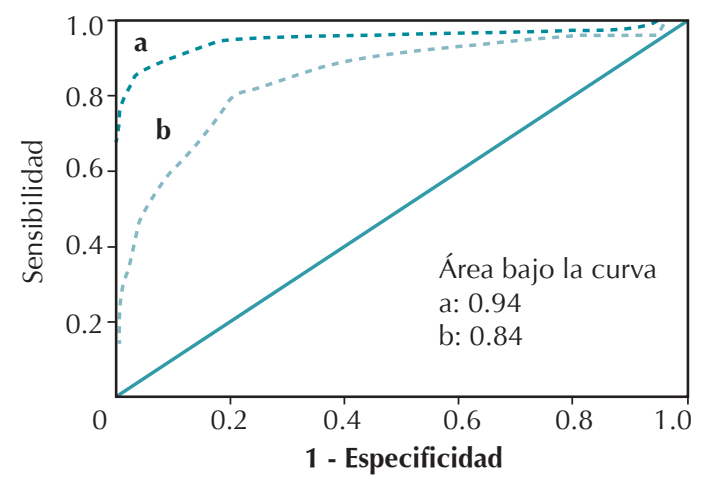

ICC

Hombres

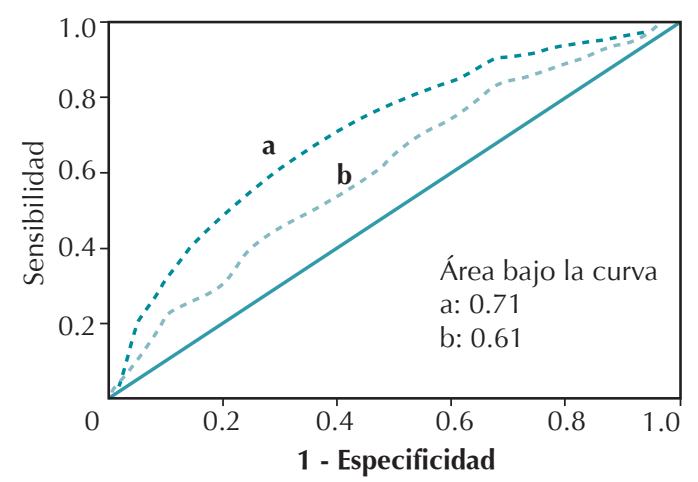

Mujeres

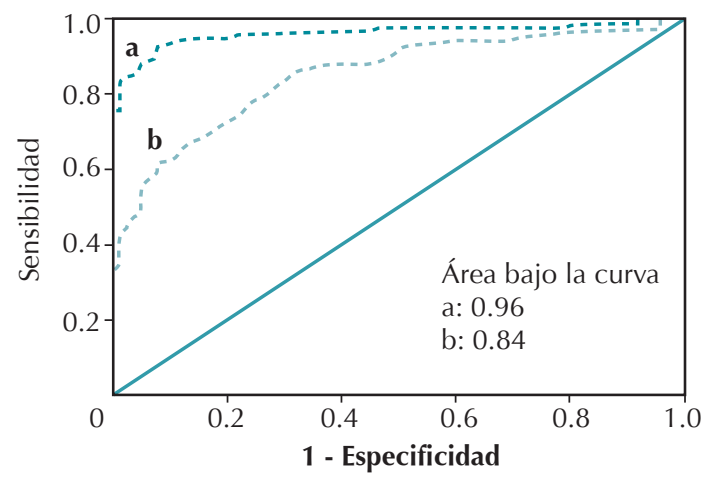

Mujeres

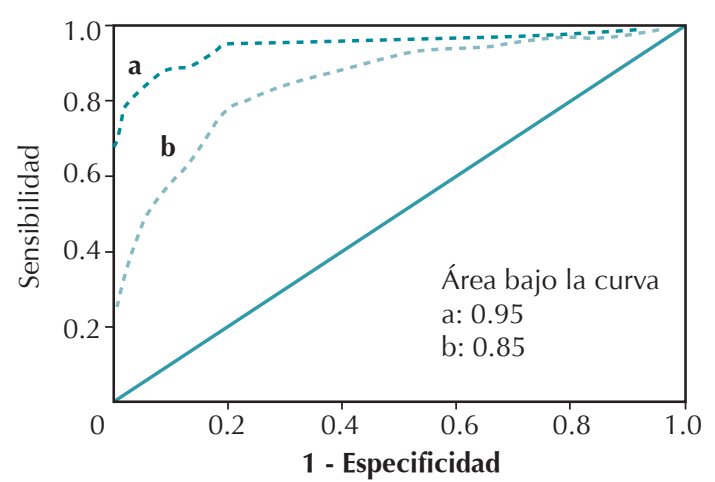

Mujeres

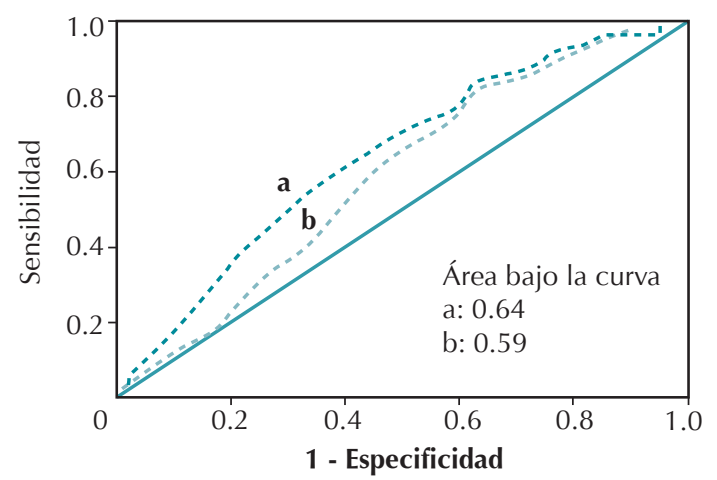

Figura 2. Curvas ROC para la identificación de obesidad (a), sobrepeso (b) por medio del perímetro de cintura (PC), índice cintura-talla (ICT), índice cintura-cadera (ICC), usando el índice de masa corporal como parámetro de referencia. A la izquierda el grupo de hombres y a la derecha el grupo de mujeres. Todas las pruebas fueron significativas $(\mathrm{p}<0.001)$. 
Saldívar-Cerón HI et al. Indicadores antropométricos de obesidad infantil

Cuadro3. Punto de corte de los indicadores antropométricos para la identificación de obesidad en escolares de 9-11 años

\begin{tabular}{|c|c|c|c|c|c|c|c|}
\hline & ABC (IC 95\%) & Punto de corte & Sens. $(\%)$ & Espec. (\%) & ES & VPP (\%) & VPN (\%) \\
\hline \multicolumn{8}{|c|}{ Hombres } \\
\hline PC & $0.96(0.93-0.97) *$ & $70 \mathrm{~cm}$ & 91.4 & 92.2 & 0.01 & 93 & 91 \\
\hline $\mathrm{ICT}$ & $0.94(0.90-0.96)^{*}$ & 0.50 & 87.7 & 96.1 & 0.01 & 94 & 94 \\
\hline ICC & $0.71(0.67-074)^{*}$ & 0.91 & 48.7 & 80.5 & 0.01 & 68 & 74 \\
\hline \multicolumn{8}{|c|}{ Mujeres } \\
\hline PC & $0.96(0.93-098)^{*}$ & $70 \mathrm{~cm}$ & 92.1 & 91.0 & 0.01 & 95 & 87 \\
\hline $\mathrm{ICT}$ & $0.95(0.91-0.97)^{*}$ & 0.50 & 83.4 & 95.1 & 0.01 & 95 & 93 \\
\hline ICC & $0.64(0.58-0.69)^{*}$ & 0.91 & 30.2 & 82.8 & 0.02 & 35 & 80 \\
\hline
\end{tabular}

ABC: área bajo la curva ROC; IC 95\%: Intervalo de confianza del 95\%; ICT: índice cintura-talla; PC: perímetro de cintura; ICC: índice cintura-cadera, Sens: sensibilidad; Espec: especificidad; ES: error estándar; VPP: valor predictivo positivo; VPN: valor predictivo negativo; ${ }^{*} \mathrm{p}<0.001$.

Cuadro 4. Punto de corte de los indicadores antropométricos para la identificación de sobrepeso en escolares de 9-11 años

\begin{tabular}{lccccccc}
\hline & ABC (IC 95\%) & Punto de corte & Sens. $(\%)$ & Espec. (\%) & ES & VPP (\%) & VPN (\%) \\
Hombres & & & & & & & \\
PC & $0.85(0.82-0.88)^{*}$ & $68 \mathrm{~cm}$ & 73.7 & 83.2 & 0.02 & 37 & 89 \\
ICT & $0.84(0.80-0.87)^{*}$ & 0.47 & 79.4 & 79.4 & 0.02 & 47 & 92 \\
ICC & $0.61(0.56-0.65)^{*}$ & 0.90 & 40.3 & 69.1 & 0.02 & 15 & 90 \\
Mujeres & & & & & & & \\
PC & $0.84(0.80-0.89)^{*}$ & $68 \mathrm{~cm}$ & 70.7 & 81.6 & 0.02 & 27 & 89 \\
ICT & $0.85(0.81-0.89)^{*}$ & 0.47 & 78.1 & 80.3 & 0.02 & 50 & 87 \\
ICC & $0.59(0.53-0.65)^{*}$ & 0.90 & 26.1 & 78.3 & 0.03 & 8 & 92
\end{tabular}

ABC: área bajo la curva ROC; IC 95\%: Intervalo de confianza del 95\%; ICT: índice cintura-talla; PC: perímetro de cintura; ICC: índice cintura-cadera; Sens: sensibilidad; Espec: especificidad; ES: error estándar; VPP: valor predictivo positivo; VPN: valor predictivo negativo; ${ }^{*} \mathrm{p}<0.001$.

índice de masa corporal para el diagnóstico de sobrepeso y obesidad infantil. ${ }^{12,13}$

El índice de masa corporal se construye a partir de mediciones sencillas (peso y talla), pero requiere la consulta de valores de referencia, por lo cual el perímetro de cintura, el índice cintura-talla y el índice cintura-cadera resultan ventajosos por su sencillez y facilidad de cálculo e interpretación. La finalidad de este estudio fue evaluar la precisión diagnóstica y los puntos de corte de esos indicadores antropométricos para la identificación de sobrepeso y obesidad infantil mexicana.

El análisis de los resultados del coeficiente de Pearson revela que el perímetro de cintura y el índice cintura-talla tienen una correlación fuertemente positiva con el índice de masa corporal a diferencia del índice cintura-cadera, lo que indica que el perímetro de cintura es un mejor predictor independiente del índice de masa corporal que cuando se combina con la talla.

En el análisis de las curvas ROC la probabilidad de identificar sobrepeso (usando cualquiera de los 3 indicadores) es baja, a diferencia de la identificación de obesidad la cual fue muy alta (exceptuando el índice cintura-cadera). Frente a los 3 indicadores, el índice cintura-talla muestra ligera ventaja al tener mayor VPP y VPN aunque su $A B C, S$ y $E$ fueron menores que los presentados por el perímetro de cintura; recordando 
que el $\mathrm{ABC}$, la $\mathrm{S}$ y la $\mathrm{E}$ son valores teóricos que no varían entre poblaciones y por tanto no son útiles en la práctica por sí solos. En cambio, el VPP y VPN evalúan el comportamiento de una prueba diagnóstica y valoran la relevancia de su $\mathrm{S}$ y E en una población determinada. ${ }^{14}$

Cabe señalar que el índice cintura-talla muestra estabilidad durante las fases de crecimiento, teniendo variaciones mínimas entre los 6 y 14 años a diferencia del perímetro de cintura que puede tener modificaciones durante la pubertad. ${ }^{15}$

Los valores de referencias del perímetro de cintura e índice cintura-talla en escolares no son concluyentes y hacer comparaciones es difícil. Sin embargo, Marrodán y su grupo determinaron para el índice cintura-talla un punto de corte de 0.50 en el diagnóstico de obesidad idéntico al reportado por este estudio; ${ }^{9}$ no obstante, concluyeron que el punto de corte de 0.48 tiene una buena $A B C, S$ y $E$ para identificar sobrepeso a diferencia de los resultados del presente estudio.

Algunos autores han calculado diversos puntos de corte para identificar obesidad infantil y su correlación con factores de riesgo cardiovascular, entre ellos Kuba y sus colaboradores ${ }^{16}$ que reportaron que el índice cintura-talla con un punto de corte $>0.47$ fue sensible para la detección de resistencia a la insulina, además de presentar más de un criterio de síndrome cardiometabólico. Algunos resultados similares fueron publicados por Muñoz y su equipo ${ }^{17}$ al identificar que el índice cintura-talla $>0.5$ se asocia con la presencia de factores de riesgo cardiovascular. En México se han determinado percentiles para identificar obesidad en una población de 8-9 años, publicando puntos de corte más altos que los de esta investigación. ${ }^{18}$ Con respecto al perímetro de cintura Maffeis y sus colegas ${ }^{19}$ reportaron que independientemente de identificar obesidad, el punto de corte $>90 \mathrm{~cm}$ representa alteración en el perfil de lípidos e hipertensión arterial sistémica en escolares; de igual manera Perichart y su grupo ${ }^{20}$ reportan que un perímetro de cintura $>80 \mathrm{~cm}$ es un indicador de hipertensión arterial sistémica, resistencia a la insulina e hipertrigliceridemia.

Por otro lado, los puntos de corte para identificar obesidad infantil no son homogéneos debido a los cambios en la distribución de grasa corporal durante el crecimiento y en diferentes poblaciones y estilos de vida tal como reportan Liu y su equipo $^{21}$ en un estudio en China determinando puntos de corte por arriba (perímetro de cintura $>75 \mathrm{~cm}$ ) de los antes mencionados.

Sin embargo, la metodología seguida en este estudio y el amplio tamaño de la muestra permiten validar el perímetro de cintura y el índice cintura-talla como indicadores precisos de obesidad infantil, empero, la muestra del presente estudio tiene como limitante observar resultados válidos sólo para la población mexicana de 9 a 11 años y consideramos que es necesario calcular los puntos de corte para toda la población escolar.

\section{CONCLUSIÓN}

Para la identificación de obesidad infantil en mexicanos entre 9 y 11 años se proponen para su empleo el perímetro de cintura y el índice cintura-talla con un punto de corte de $70 \mathrm{~cm} \mathrm{y}$ 0.5 , respectivamente. No obstante, debido a que el patrón de distribución de la grasa difiere en función del origen poblacional, ${ }^{22}$ el uso de estos puntos de corte no debe extrapolarse a grupos humanos de distinto origen étnico.

\section{Agradecimientos}

El más cordial agradecimiento al Dr. Enrique Álvarez Viaña y a la Dra. Leticia Manuel Apolinar, sin su ayuda este proyecto no se hubiera podido llevar a cabo. 
Saldívar-Cerón HI et al. Indicadores antropométricos de obesidad infantil

\section{REFERENCIAS}

1. Obesity: preventing and managing the global epidemic. Report of a WHO consultation. World Health Organization technical report series. 2000;894:i-xii, 1-253.

2. Amato $M$, Giordano $C$, Pitrone $M$, Galluzzo A. Cut-off points of the visceral adiposity index (VAl) identifying a visceral adipose dysfunction associated with cardiometabolic risk in a Caucasian Sicilian population. Lipids in Health and Disease. 2011;10(1):183.

3. Neovius M, Linne $\mathrm{Y}$, Rossner S. BMI, waist-circumference and waist-hip-ratio as diagnostic tests for fatness in adolescents. International journal of obesity. 2005;29(2):163-9.

4. Ryo M, Kishida K, Nakamura T, Yoshizumi T, Funahashi T, Shimomura I. Clinical significance of visceral adiposity assessed by computed tomography: A Japanese perspective. World Journal of Radiology. 2014;6(7):409-16.

5. Duelo MM, Escribano CE, Muñoz VF. Obesidad. Rev Pediatr Aten Primaria. 2009;11(16):239-57.

6. de Onis M. Preventing childhood overweight and obesity. J Pediatr. 2015;91(2):105-7.

7. Kuba VM, Leone C, Damiani D. Is waist-to-height ratio a useful indicator of cardio-metabolic risk in 6-10-year-old children? BMC pediatrics. 2013;13:91.

8. Pérez BM, Landaeta-Jiménez M, Amador J, Vásquez M, Marrodán $\mathrm{D}$. Sensibilidad y especificidad de indicadores antropométricos de adiposidad y distribución de grasa en niños y adolescentes Venezolanos. Interciencia. 2009;34:84-90.

9. Marrodan MD, Martinez-Alvarez JR, Gonzalez-Montero De Espinosa M, Lopez-Ejeda N, Cabanas MD, Prado C. Diagnostic accuracy of waist to height ratio in screening of overweight and infant obesity. Medicina clinica. 2013;140(7):296-301.

10. Saldívar-Ceron HI, Garmendia RA, Rocha AMA, Pérez-Rodríguez P. Obesidad infantil: factor de riesgo para desarrollar pie plano. Bol Med Hosp Infant Mex. 2015;72(1):55-60.

11. Sung RY, Yu CC, Choi KC, McManus A, Li AM, Xu SL, et al. Waist circumference and body mass index in Chinese children: cutoff values for predicting cardiovascular risk factors. International journal of obesity. 2007;31(3):550-8.

12. Van den Broeck J, Willie D, Younger N. The World Health Organization child growth standards: expected implications for clinical and epidemiological research. European journal of pediatrics. 2009;168(2):247-51.
13. Sanchez Gonzalez E, Carrascosa Lezcano A, Fernandez Garcia JM, Ferrandez Longas A, Lopez de Lara D, Lopez-Siguero JP. Spanish growth studies: the current situation, their effectiveness and recommendations for their use. Anales de pediatria (Barcelona, Spain : 2003). 2011;74(3):193. e1-16.

14. Altman DG, Bland JM. Statistics Notes: Diagnostic tests 2: predictive values1994 1994-07-09 07:00:00. $102 \mathrm{p}$.

15. Mihalopoulos NL, Holubkov R, Young P, Dai S, Labarthe DR. Expected changes in clinical measures of adiposity during puberty. The Journal of adolescent health : official publication of the Society for Adolescent Medicine. 2010;47(4):360-6.

16. Kuba VM, Leone C, Damiani D. Is waist-to-height ratio a useful indicator of cardio-metabolic risk in 6-10-year-old children? BMC pediatrics. 2013;13:91.

17. Muñoz-Cano JM, Pérez-Sánchez $S$, Córdova-Hernández JA, X B-L. El índice cintura/talla como indicador de riesgo para enfermedades crónicas en una muestra de escolares. . Salud en Tabasco. 2010;16:921-7.

18. Gómez-Díaz RA, Martínez-Hernández AJ, Aguilar-Salinas CA, Violante R, Alarcón ML, Villarruel MJ, et al. Percentile distribution of the waist circumference among Mexican pre-adolescents of a primary school in Mexico City. Diabetes, Obesity and Metabolism. 2005;7(6):716-21.

19. Maffeis C, Pietrobelli A, Grezzani A, Provera S, Tato L. Waist circumference and cardiovascular risk factors in prepubertal children. Obesity research. 2001;9(3):179-87.

20. Perichart-Perera O, Balas-Nakash M, Schiffman-Selechnik E, Barbato-Dosal A, Vadillo-Ortega F. Obesity increases metabolic syndrome risk factors in school-aged children from an urban school in Mexico city. Journal of the American Dietetic Association. 2007;107(1):81-91.

21. Liu A, Hills AP, Hu X, Li Y, Du L, Xu Y, et al. Waist circumference cut-off values for the prediction of cardiovascular risk factors clustering in Chinese school-aged children: a cross-sectional study. BMC public health. 2010;10:82.

22. Romero-Collazos JF, Marrodán Serrano MD, Mesa Santurino MS, Bejarano I, Alfaro E, Martínez A. Grasa corporal y distribución de la adiposidad en escolares latinoamericanos y españoles. En: Gutiérrez-Redomero A, Sánchez-Andrés A, Galera Olmo V, editores. Diversidad Humana y Antropología Aplicada. Alcalá de Henares: Universidad de Alcalá de Henares. 2010. p. 221-7. 